\title{
Resonant absorption enhancement in solar cells with periodically textured interfaces
}

\author{
Franz-Josef Haug, Karin Söderström, Christophe Ballif \\ Ecole Polytechnique Fédérale de Lausanne (EPFL), Institute of Microengineering, Photovoltaics and Thin Film Electronics \\ Laboratory, Rue A.-L. Breguet 2, CH-2000 Neuchâtel, Switzerland
}

\begin{abstract}
We use a $1 \mathrm{D}$ grating back reflector to study the mechanism of light trapping in solar cells. Experimental results of a full solar cell on a sinusoidal back reflector are compared to a theoretical model using an expansion into grating modes and the Rayleigh hypothesis. By means of the sinusoidal shape, the continuity equations extend the coupling only to neighboring orders. The essential aspects of the coupling process are thus included, but the algorithm requires only a minimum amount of mathematical complexity. Our modeling results correctly reproduce the absorption of the periodically textured solar cell for both polarization directions. The absorption phenomena underlying the light trapping process can thus be studied with a small number of parameters like period, depth, and film thickness.
\end{abstract}

Index Terms - amorphous silicon, light trapping, diffraction efficiency

\section{INTRODUCTION}

Interface textures are used in various electronic components to enhance the coupling between the active layers and external radiation. Prominent examples are emission enhancement in light emitting diodes and absorption enhancement in detectors and in solar cells. The latter is most widely studied in devices based on thin film silicon but could become an interesting option to reduce the film thickness in other solar cell technologies. Owing to their ease of fabrication, the most successful light scattering designs rely on random textures that were proposed as early as 1983 by Deckman [1]. However, already then it was suggested that cleverly designed periodic interface textures should outperform their random counterparts [2]. In the meantime, promising device efficiencies have been demonstrated for devices with periodic textures [3-7], but performance going beyond random interface texture has not yet been demonstrated convincingly. With advancing computing power, rigorous calculations have become accessible to the modeling of solar cells [8-10], but the understanding of the actual mechanisms of light trapping is still limited.

In this contribution, we study absorption phenomena in solar cell structures with one-dimensional interface texture. The reduced complexity of the device design gives distinguishing the key parameters underneath the light trapping process, especially the excitation of waveguide modes.

\section{EXPERIMENTAL}

The experimental results were carried out on a sinusoidal grating with period of $550 \mathrm{~nm}$ and amplitude of $70 \mathrm{~nm}$ (140 nm peak-to-valley depth). The solar cell was deposited in n-i-p configuration using a stack of ITO/a-Si/ZnO/Ag with respective thicknesses of 65/255/60/120 nm [6]. ITO, ZnO and $\mathrm{Ag}$ were deposited by sputtering. The $255 \mathrm{~nm}$ thick silicon film contains $n$ - and p-doped layers with respective thicknesses of 20 and $10 \mathrm{~nm}$, respectively, all deposited by plasma enhanced chemical vapor deposition with an excitation frequency of $70 \mathrm{MHz}$.

The reflection was measured from 370 to $2000 \mathrm{~nm}$ in a spectrophotometer with integrating sphere which yields an angle of incidence of $7^{\circ}$ (Lambda 900, Perkin Elmer). The measurement on the small cell surface required reduced spot size and becomes thus somewhat noisy towards the boundaries of the investigated spectral region. A broad band wire grid polarizer (ProFlux PPL05C, Moxtek) was used to polarize the incident beam parallel or perpendicular to the grating lines, exciting thus TE or TM modes, respectively.

\section{MODEL}

We expand the electromagnetic field of each layer into forward and backward traveling grating modes. The delimiting half spaces must be treated separately. We assume a loss-free incident medium a refractive index of unity and we express the incident light by a single plane-wave. The reflected light contains propagating and evanescent grating modes which are described by purely real and purely imaginary wave vectors, respectively. Since the silver back-electrode is opaque, we do not take into account its actual thickness, but we assume that it fills the opposing half-space. It contains thus only forward travelling waves that are transmitted from the other layers into the silver film. In the layers between the air- and silver-filled half-spaces, the forward and backward going wave vectors are generally complex which denotes propagation with varying amounts of attenuation.

In order to evaluate the continuity conditions at the interfaces, we make use of the Rayleigh hypothesis, i.e. we assume that the expansion into diffraction modes remains valid in the roughness zones and at the interfaces. While not applicable in general, the Rayleigh hypothesis was reported to apply reliably for gratings similar to ours [11]. Application of the continuity equations yields the normal derivative of the surface profile. Here, the sinusoidal shape yields one of the simplest possible coupling mechanisms; its spatial Fouriertransform contains only a single frequency and couples thus only to neighboring orders [12]. The expansion is subsequently sorted by the orders of the diffraction modes and 
truncated to desired accuracy. The procedure maintains the coupling in a mathematically rigorous manner and shows fast convergence, typically for three or four orders $[11,13,14]$.

\section{RESULTS AND DISCUSSION}

We applied the model to the solar cell described in ref. [6]. All modeling results were obtained with truncation after third order. Figs. 1 and 2 show the total reflection in TE- and TM polarization, respectively. In both figures, Fabri-Perot interference yields features that are common in both polarization directions. They are easily identified by either truncating the series after zero order, or by reducing the grating depth to zero. Both methods represent a flat structure where maxima appear at 1450, 920, 700, and at $575 \mathrm{~nm}$ (not shown). The presence of the grating yields blue-shifts to 1300 , 870,680 and $555 \mathrm{~nm}$, respectively.

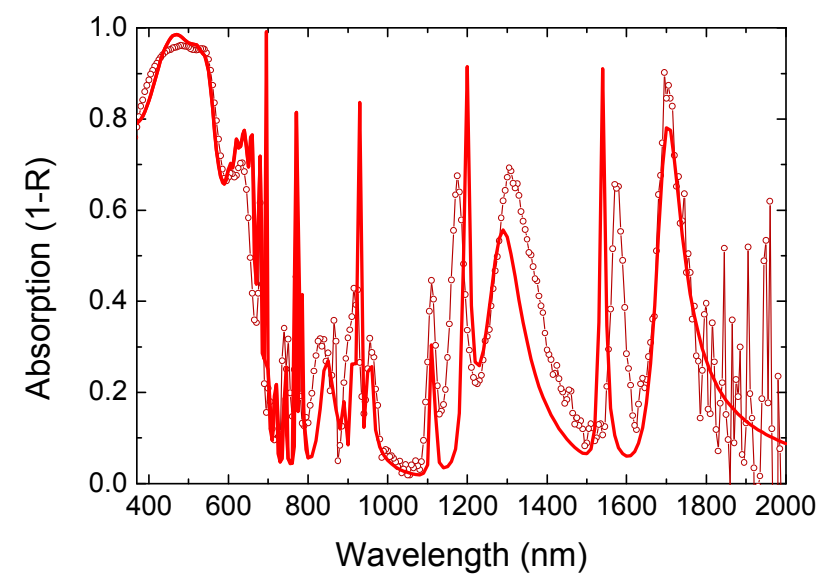

Fig. 1: Total reflection of the solar cell in TE-polarization over a wide spectral region (symbols). The lines illustrate modeling results.

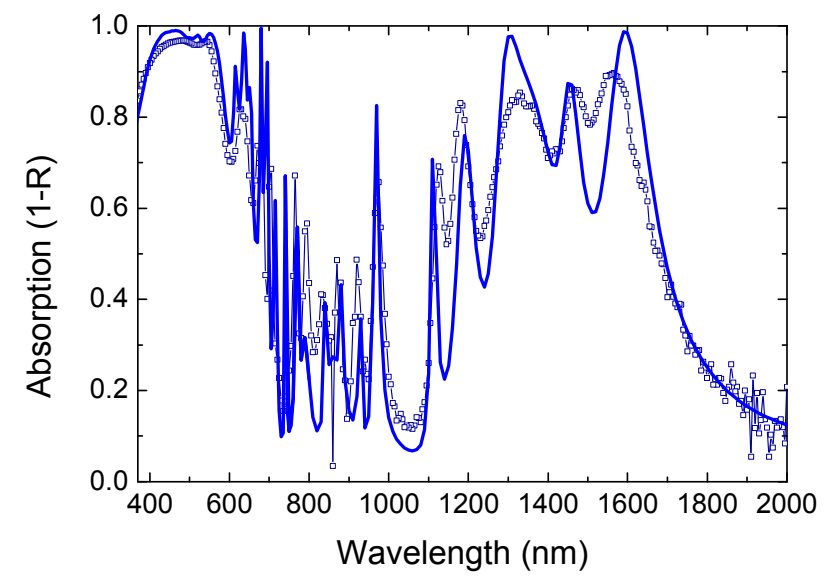

Fig. 2: Total reflection of the solar cell in TM-polarization over a wide spectral region (symbols). The lines illustrate modeling results.

Between the Fabri-Perot interferences, Figs. 1 and 2 show a multitude of guided modes, notably the broad absorption signatures at $1700 \mathrm{~nm}$ and around $1500 \mathrm{~nm}$ found for TE and TM polarization, respectively. The modeling results denoted by the lines show a good overall correspondence to the measured results, all of the dominant features of the measurement are correctly reproduced. A closer investigation of Figs. 1 and 2 shows slight misfits of the peak positions, notably around 1200 and $1600 \mathrm{~nm}$. Similarly, the height and width of the peaks show slight deviations. These two observations are most likely related to our assumption of perfectly reproduced interfaces whereas sputtering and CVD processes are known to deviate from fully conformal growth.

Figs. 3 and 4 zoom in on the range of interest for solar cell operation. For both polarization directions, the high absorption between 400 and $550 \mathrm{~nm}$ is related to the thickness of the ITO front electrode. Its thickness of $65 \mathrm{~nm}$ is designed to yield an anti-reflection condition close to $500 \mathrm{~nm}$ (c.f. characteristic of flat structure in Figs. 3 and 4). The interface texture enhances the anti-reflection effect towards shorter wavelengths.

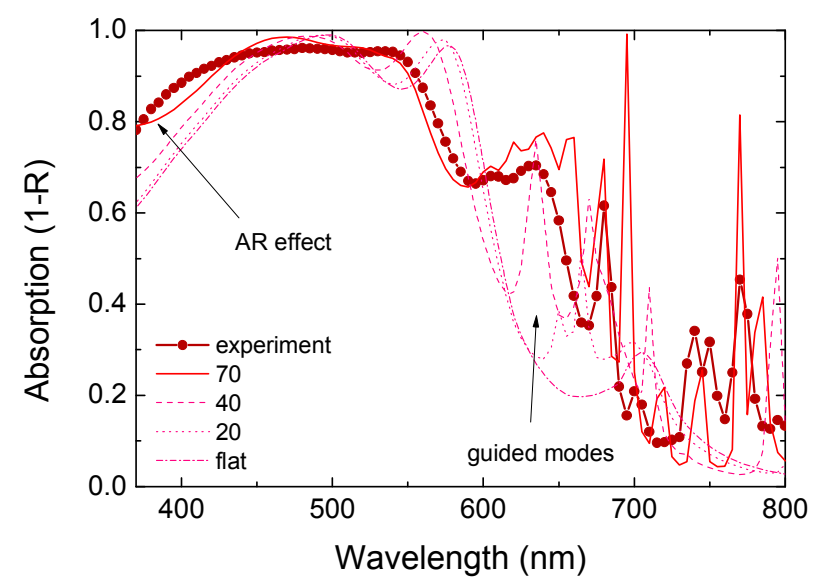

Fig. 3: Detail of the absorption characteristic in TE polarization. The full line denotes the grating amplitude of 70 used in the experiment, dashed and dotted lines refer to grating amplitudes of 40 and $20 \mathrm{~nm}$, respectively, and the and dash-dotted line illustrates the properties of a flat structure.

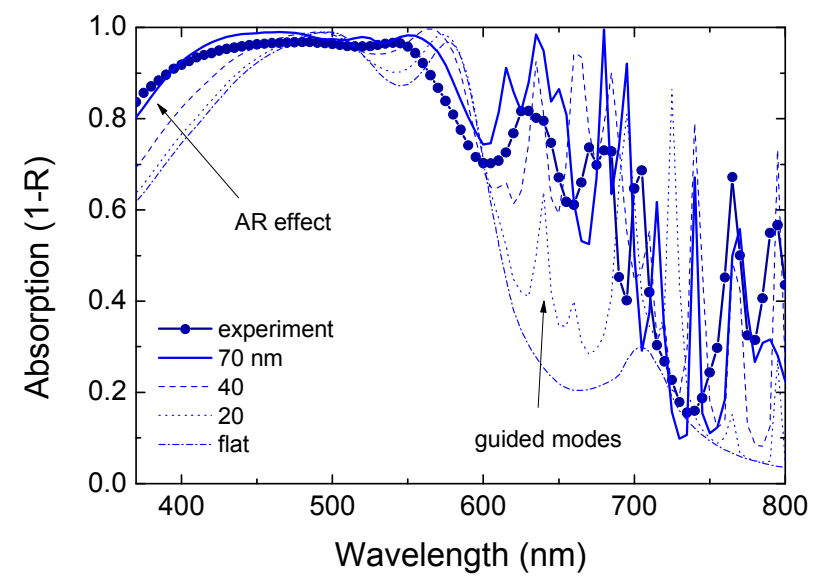

Fig. 4: Detail of the absorption characteristic in TM polarization. The line styles are the same as Fig. 3.

Increasing grating amplitudes yield a blue shift of the dominant Fabri-Perot interference at $570 \mathrm{~nm}$. Eapecially for 
TE polarization a sudden drop of absorption appears as the amplitude is stepped up from 40 to $70 \mathrm{~nm}$.

In the light trapping region between 600 and $750 \mathrm{~nm}$, increasing grating amplitude yields more and more efficient coupling to guided modes. The experimental data do not resolve all of the narrow resonances that dominate the modeling results in this region, but absorption enhancement is clearly visible for both polarization directions. Overall, the enhancement appears to be more efficient for TM-polarization shown in Fig. 4.

Since the model gives direct access to the electromagnetic field amplitudes within each of the layers, we plan to extract their individual absorption signatures. We will then compare the absorption inside the intrinsic region with measurements of the external quantum efficiency [6] and we will identify parasitic absorption channels in the supporting films like the doped layers and the metal back contact.

\section{ACKNOWLEDGEMENT}

We thankfully acknowledge funding by Swiss National Science Foundation (No. 200020_137700/1) and by the European Union within the projects Silicon-Light (No. 241277) and FastTrack (No. 283501).

\section{REFERENCES}

[1] H. W. Deckman, et al., "Optically enhanced amorphous silicon solar cells", Applied Physics Letters 42(11), p. 968970 (1983).

[2] P. Sheng, A. N. Bloch, and R. S. Stepleman, "Wavelengthselective absorption enhancement in thin-film solar cells", Applied Physics Letters 43, p. 579 (1983).

[3] N. Senoussaoui, et al., "Thin-film solar cells with periodic grating coupler", Thin Solid Films 451, p. 397-401 (2004).

[4] V. E. Ferry, et al., "Improved red-response in thin film a-Si: H solar cells with soft-imprinted plasmonic back reflectors", Applied Physics Letters 95, p. 183503 (2009).
[5] J. Zhu, et al., "Nanodome solar cells with efficient light management and self-cleaning", Nano Letters 10(6), p. 1979-1984 (2009).

[6] K. Söderström, et al., "Photocurrent increase in n-i-p thin film silicon solar cells by guided mode excitation via grating coupler", Applied Physics Letters 96, p. 213508 (2010).

[7] U. Paetzold, et al., "Plasmonic reflection grating back contacts for microcrystalline silicon solar cells", Applied Physics Letters 99(18), p. 181105 (2011).

[8] C. Haase and H. Stiebig, "Thin-film silicon solar cells with efficient periodic light trapping texture", Applied Physics Letters 91(6), p. 061116 (2007).

[9] C. Rockstuhl, et al., "Light localization at randomly textured surfaces for solar-cell applications", Applied Physics Letters 91, p. 171104 (2007).

[10] A. Campa, et al., "Optimal design of periodic surface texture for thin film a-Si:H solar cells", Progress in Photovoltaics: Research and Applications 18(3), p. 160-167 (2010).

[11] S. H. Zaidi, M. Yousaf, and S. R. J. Brueck, "Grating coupling to surface plasma waves. I. First-order coupling", Journal of the Optical Society of America B 8(4), p. 770779 (1991).

[12] V. Kiselev, "Resonant conversion and reflection of surface waves in a thin-film waveguide with a sinusoidally corrugated surface", Soviet Journal of Quantum Electronics 4, p. 182 (1974).

[13] S. H. Zaidi, et al., "Characterization of thin Al films using grating coupling to surface plasma waves", Journal of Applied Physics 71(12), p. 6039-6048 (1992).

[14] F. J. Haug, A. Naqavi, and C. Ballif, "Diffraction and absorption enhancement from textured back reflectors of thin film solar cells ", Journal of Applied Physics 112, p. 024516 (2012). 